\title{
Expression of metabotropic glutamate receptor 4 in osteosarcoma
}

\author{
SHUO WANG ${ }^{1}$, XING WEI $^{1}$, BINGYAO CHEN ${ }^{1}$, MIN ZHAO $^{2}$, \\ GUANGZE SONG $^{1}$, ZENGLIANG ZHANG ${ }^{1}$ and NAN LI ${ }^{1}$ \\ Departments of ${ }^{1}$ Orthopedic Oncology and ${ }^{2}$ Pathology, the First Affiliated Hospital of Chinese \\ People's Liberation Army General Hospital, Beijing 100048, P.R. China
}

Received June 15, 2015; Accepted October 13, 2015

DOI: $10.3892 / \mathrm{mco} .2015 .661$

\begin{abstract}
Metabotropic glutamate receptor 4 (mGluR4) has been associated with the pathogenesis of osteosarcoma. The aim of this study was to investigate mGluR4 expression and its clinical significance in osteosarcoma patients. mGluR4 expression was investigated using immunohistochemistry (IHC) in 58 osteosarcomas and 32 giant-cell tumors of bone. The correlations between mGluR4 expression and clinicopathological characteristics were analyzed with the Chi-squared test and survival curves were generated using the Kaplan-Meier method. The IHC results demonstrated that $20.69 \%(12 / 58)$ of the osteosarcomas and $43.75 \%(14 / 32)$ of the giant-cell tumors were mGluR4-positive. The statistical analysis revealed that mGluR4 expression was correlated with gender, age, Enneking stage and tumor volume in osteosarcomas $(\mathrm{P}<0.05)$. In the multivariate stepwise Cox regression analysis, Enneking stage was found to be statistically significantly associated with survival $(\mathrm{P}<0.05)$ and the survival analysis demonstrated that the survival probability was significantly higher in patients with higher mGluR4 expression compared with those with lower expression $(\mathrm{P}<0.05)$. Therefore, mGluR4 expression may be used to estimate the prognosis of osteosarcoma patients.
\end{abstract}

\section{Introduction}

Osteosarcomas are the most common primary musculoskeletal malignant tumors in children, accounting for $~ 5 \%$ of all pediatric tumors (1). Conventionally, chemotherapy has been used to improve patient survival; however, this therapeutic approach has reached a plateau in the last two decades. Over the last decade, targeted therapy was introduced and has achieved great success in various malignancies, such as leukemia, melanoma, and lung and breast cancer. However, patients with

Correspondence to: $\mathrm{Dr} \mathrm{Nan} \mathrm{Li}$, Department of Orthopedic Oncology, the First Affiliated Hospital of Chinese People's Liberation Army General Hospital, 51 Fucheng Street, Haidian, Beijing 100048, P.R. China

E-mail: orthopnanli@gmail.com

Key words: metabotropic glutamate receptor 4, immunohistochemistry, osteosarcoma, prognosis osteosarcoma have not yet benefited from targeted therapy, due to the fact that the genetic etiology of osteosarcomas is complex and has not been fully elucidated, with no specific therapeutic targets yet identified.

To better understand the genetic etiology of osteosarcomas, one recent genome-wide association study (GWAS) investigated 941 patients with osteosarcoma and 3,291 cancer-free adult controls of European descent (2). That study identified two significant loci, one located at $6 \mathrm{p} 21.3$, containing the GRM4 gene, which encodes the metabotropic glutamate receptor 4 (mGluR4), whereas the other one was located in the gene desert. The study suggested that mGluR4 may play an important role in the pathogenesis of osteosarcoma. The amino acid glutamate is a fundamental extracellular messenger in a number of tissues and functions in neural and non-neural signaling in bone to maintain structural and functional homeostasis. Glutamic acid signals through cell surface glutamate receptors, which have been classified into two types, ionotropic glutamate receptors (iGluRs) and mGluRs. iGluRs, the first structure identified in the glutamic acid receptor family, are classic ligand-gated ion channels, including $\mathrm{N}$-methyl-D-aspartate and $\alpha$-amino-3-hydroxy-5methylisoxazole-4-propionate/kainate receptors (3), which allow cations such as $\mathrm{Na}^{+}$and $\mathrm{Ca}^{2+}$ to enter the cell. By contrast, mGluRs are G-protein-coupled receptors that stimulate secondary messengers, such as inositol triphosphate (IP3), diacylglycerol (DAG) and cAMP to generate the desired signaling effect (4).

Accumulating evidence has revealed that mGluRs are involved in cancer pathophysiology, with their mRNA detected in various cancer cell lines (5). However, the number of studies investigating the physiological roles of $\mathrm{mGulRs}$ in sarcomas is currently limited. In this preliminary study, mGluR4 expression in osteosarcoma tissues and its correlations with clinical characteristics were assessed.

\section{Patients and methods}

Patients and tissue specimens. The specimens were collected retrospectively. This study was approved by the Internal Review Board of the First Affiliated Hospital of PLA General Hospital (Beijing, China) and all the pathological specimens (osteosarcomas, giant-cell tumors and one cerebellar tissue sample) were obtained from the hospital's Department of Pathology database. A total of 58 osteosarcoma samples 
(35 from male and 23 from female patients) and 32 giant-cell tumor of bone samples (20 from male and 12 from female patients) were collected and reviewed by two pathologists. All the samples originated from definitive surgeries, apart from 2 osteosarcoma samples derived from biopsies. Osteosarcoma patient demographics, such as age, gender, tumor location, histological subtype, metastasis and Enneking stage, were analyzed. In osteosarcoma patients, the mean age at presentation was 19.7 years (range, 5-61 years) and the tumor sites included the femur $(n=32)$, tibia and fibula $(n=16)$, humerus $(n=6)$, scapula $(n=2)$, rib $(n=1)$ and sternum $(n=1)$. The histological subtypes included osteoblastic $(n=42)$, chondroblastic $(n=12)$, fibroblastic $(n=3)$ and small-cell $(n=1)$ osteosarcomas. All the patients were regularly followed up, with a mean of 30.9 months (range, 12-102 months) from 2006 to 2014. The diagnoses were confirmed by histological analysis and radiographic findings in all the cases; the histological characteristics included spindle cell proliferation in osteosarcoma, and the presence of multinucleated giant cells in giant-cell tumors. Cerebellar tissue was used as a control for mGRM4 expression evaluated via immunohistochemistry.

Immunohistochemistry. The specimens were fixed in formalin, decalcified in 5\% nitric acid for $12 \mathrm{~h}$, embedded in paraffin and sectioned $(2 \mu \mathrm{m})$. The sections were dewaxed and hydrated, with a heat-antigen retrieval method used prior to incubation with $0.01 \mathrm{M}$ sodium citrate buffer solution (pH 6.0) for $10 \mathrm{~min}$ at $90^{\circ} \mathrm{C}$. The sections were then incubated in $3 \%$ hydrogen peroxide for $10 \mathrm{~min}$ at $37^{\circ} \mathrm{C}$ to inactivate endogenous peroxidase, followed by incubation with a drop of goat serum [1:10 with phosphate-buffered saline (PBS)] for $1 \mathrm{~h}$ at $37^{\circ} \mathrm{C}$. Each section was then stained with mouse monoclonal anti-mGluR4 antibody (cat. no. sc-376485; 1:100; Santa Cruz Biotechnology, USA) and incubated overnight at $4^{\circ} \mathrm{C}$. Subsequently, the sections were incubated with biotin-labeled goat anti-mouse secondary antibody (cat. no. ZB-2305; 1:100; Zhongshan Biotechnology, Beijing, China) for $1 \mathrm{~h}$ and colorimetrically detected with horseradish peroxidase-labeled avidin-biotin complex and diaminobenzidine (Zhongshan Biotechnology) at $37^{\circ} \mathrm{C}$ for $7 \mathrm{~min}$. The sections were rinsed 3-5 times with PBS between steps, with the exception of the last step. The cerebellar tissue was stained in the same manner with anti-mGluR4 antibodies and hematoxylin, and displayed a positive result; it was previously demonstrated that mGluR4 is present in cerebellar granule cell neuroprogenitors (6).

The immunostaining results were independently evaluated by two pathologists (Min Zhao and Yiduo Jin) who were blinded to the clinical data; any discrepancies were resolved by consensus. The result was calculated as the percentage of positively stained cells, with only cytoplasmic mGluR4 staining considered as a positive result. Specimen staining was defined based on positivity or negativity for mGluR4. Immunostaining positivity was defined as a staining proportion of tumor cells of $\geq 10 \%$; when the staining proportion was $<10 \%$, immunostaining was defined as negative. Only strong staining intensity was considered as positive.

Statistical analysis. All the analyses were conducted using the SPSS statistical software package, version 19.0 (IBM SPSS, Armonk, NY, USA). The Chi-squared test was used to analyze differences between classified variables, with $\mathrm{P}<0.05$ deemed as statistically significant. A multivariate Cox regression analysis was performed, including the covariables with a P-value of $\leq 0.05$ in the Chi-squared test. The Kaplan-Meier method and log-rank test were used to calculate survival probability.

\section{Results}

Expression of mGluR4 in osteosarcoma and giant-cell tumors. mGlu4 expression as detected by immunohistochemical staining was examined via imaging with a BX 50-32 scanner (Olympus, Union City, CA, USA) and analyzed with NIS-Elements D software (Nikon BX Series Eclipse Ci-E; Nikon, Tokyo, Japan). As a positive control, a specimen from the cerebellum of a patient with epilepsy was used, displaying cytoplasmic mGluR4 expression in the supranuclear portion. Subsequently, mGluR4 expression was investigated in 58 osteosarcoma specimens, with $12 / 58$ staining positive (20.69\%, Fig. 1), and 32 giant-cell tumors, with 14/32 staining positive (43.75\%, Fig. 2$)$.

Association of mGluR4 expression with clinicopathological characteristics in osteosarcoma. Only cytoplasmic staining was considered to be a positive result. The correlations of clinicopathological characteristics (gender, age, tumor size, tumor location, histological type and Enneking stage) with mGluR4 expression in osteosarcoma are summarized in Table I. mGluR4 expression was correlated with gender $(\mathrm{P}=0.0308)$, age $(\mathrm{P}=0.0489)$, Enneking stage $(\mathrm{P}=0.0415)$ and tumor volume $(\mathrm{P}=0.02)$; there was no significant correlation with tumor location $(\mathrm{P}=0.9486)$ or histological type $(\mathrm{P}=0.7030)$. In the multivariate Cox regression analysis, Enneking stage exerted a statistically significant effect on survival $(\mathrm{P}<0.001)$. Furthermore, mGluR4 immunostaining and tumor volume did not exert a statistically significant effect on survival ( $\mathrm{P}=0.092$ and 0.789 , respectively). The detailed results of the Chi-squared test and multivariate analysis are summarized in Tables I and II, respectively.

Prognostic value of mGluR4 for patients with osteosarcoma. To elucidate whether mGluR4 signaling affects patient prognosis, mGluR4 expression and patient survival were investigated using the Kaplan-Meier survival analysis. Of the 12 osteosarcoma patients with positive mGluR4 expression, 11 survived, while 19 of the 46 patients with negative mGluR4 expression succumbed to the disease. Furthermore, the log-rank analysis revealed a higher survival rate in osteosarcoma patients with positive mGluR4 expression compared with those with negative expression ( $\mathrm{P}=0.0122$, Fig. 3 ). These findings suggest that mGluR4 positivity is correlated with a favorable prognosis in patients with osteosarcoma.

\section{Discussion}

There is an urgent need for identifying more osteosarcoma biomarkers. The implication of mGluR4 in osteosarcoma was revealed by a GWAS study (2). Therefore, the present study was conducted to investigate the role of mGluR4 in osteosarcoma development and prognosis. mGluR1 was initially discovered 
Table I. Expression of mGluR4 according to clinicopathological characteristics in osteosarcoma.

\begin{tabular}{|c|c|c|c|c|}
\hline \multirow[b]{2}{*}{ Characteristics } & \multicolumn{3}{|c|}{ Expression of mGluR4 } & \multirow[b]{2}{*}{$\mathrm{P}$-value } \\
\hline & $\begin{array}{c}\text { Number of } \\
\text { cases }(n=58)\end{array}$ & $\begin{array}{c}\text { Negative } \\
(n=46)\end{array}$ & $\begin{array}{c}\text { Positive } \\
(n=12)\end{array}$ & \\
\hline Gender & & & & 0.0308 \\
\hline Male & 35 & 24 & 11 & \\
\hline Female & 23 & 22 & 1 & \\
\hline Age, years & & & & 0.0489 \\
\hline$<18$ & 36 & 32 & 4 & \\
\hline$\geq 18$ & 22 & 14 & 8 & \\
\hline Location & & & & 0.9486 \\
\hline Femur & 32 & 26 & 6 & \\
\hline Tibia and fibula & 16 & 12 & 4 & \\
\hline Humerus & 6 & 5 & 1 & \\
\hline Other $^{\mathrm{a}}$ & 4 & 3 & 1 & \\
\hline Histological subtype & & & & 0.7030 \\
\hline Osteoblastic & 42 & 32 & 10 & \\
\hline Chondroblastic & 12 & 10 & 2 & \\
\hline Small-cell & 1 & 1 & 0 & \\
\hline Fibroblastic & 3 & 3 & 0 & \\
\hline Enneking stage & & & & 0.0415 \\
\hline II & 36 & 25 & 11 & \\
\hline III & 22 & 21 & 1 & \\
\hline Mean tumor volume, $\mathrm{cm}^{3}$ & & 306.55 & 195.13 & 0.02 \\
\hline
\end{tabular}

Table II. Multivariate Cox regression analysis.

\begin{tabular}{lccrrrrr}
\hline Factors & B & SE & Wald & df & Sig. & Exp(B) & $95.0 \%$ CI \\
\hline Gender & 0.430 & 0.503 & 0.732 & 1 & 0.392 & 0.650 & $0.243-1.743$ \\
Age & 0.017 & 0.021 & 0.656 & 1 & 0.418 & 0.983 & $0.943-1.025$ \\
Tumor volume & 0.000 & 0.001 & 0.072 & 1 & 0.789 & 1.000 & $0.998-1.003$ \\
Enneking stage & 3.096 & 0.821 & 14.209 & 1 & $<0.001$ & 22.111 & $4.420-110.601$ \\
mGluR4 immunostaining & 2.011 & 1.195 & 2.830 & 1 & 0.092 & 7.467 & $0.718-77.695$ \\
\hline
\end{tabular}

SE, standard error; df, degree of freedom; CI, confidence interval.

in mouse brain 20 years ago, with a total of 7 different subtypes identified to date (7). These subtypes are further divided into three functional subgroups based on their sequence homologies, signal transduction profiles and pharmacological properties as follows: Group I (mGluR1 and mGluR5), which is coupled to phospholipase $\mathrm{C}$ via $\mathrm{G}_{\mathrm{q} / 11}$ proteins, thus leading to phosphoinositide hydrolysis and the generation of IP3 and DAG; group II (mGluR2 and mGluR3), which negatively regulates adenylate cyclase $(\mathrm{AC})$ in a recombinant system; and group III (mGluR4, mGluR6, mGluR7 and mGluR8), which also negatively regulates $\mathrm{AC}$, reduces cAMP formation and may be activated by L-2-amino-4-phosphonobutyric acid $(4,8)$.
The function of mGluR4 has been widely investigated in the central nervous system, with the interference of glutamate signaling in pediatric CNS tumors shown to suppress tumor growth (9). In a previous study, mGluR4 expression was enhanced using $N$-phenyl-7-(hydroxyimino)cyclopropa[b] chromen-1a-carboxamide (PHCCC) and found proliferation to be inhibited, while differentiation was promoted in cerebellar granule cell neuroprogenitors (10). In agreement with those findings, the present data demonstrated that mGluR4 may promote differentiation to maintain mature cells. Furthermore, another study demonstrated that mGluR4 inhibits proliferation and promotes the differentiation of cerebellar granule cell 

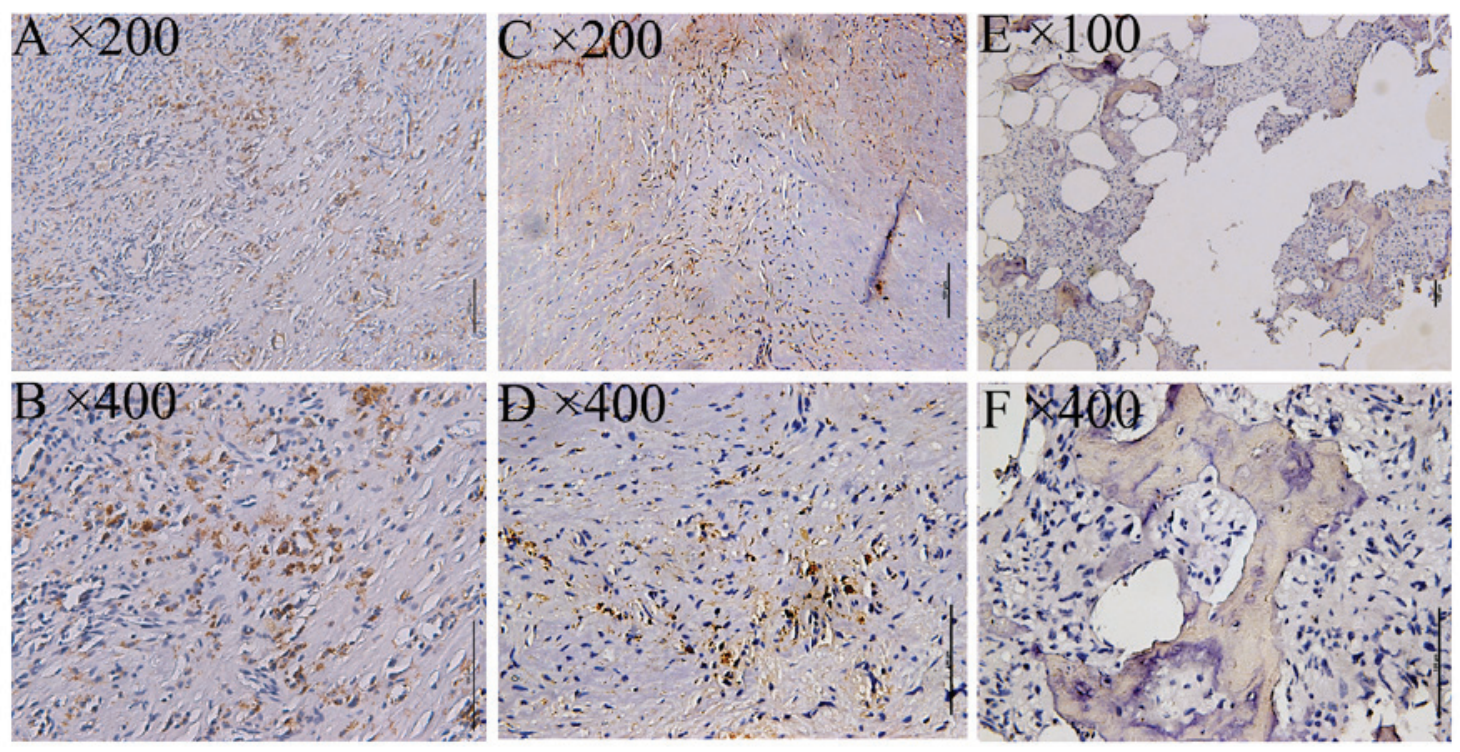

Figure 1. Immunohistochemical staining of mGluR4 in osteosarcoma. Positive staining for mGluR4 was detected as yellow to brown color in the cytoplasmic area of osteosarcoma cells. (A and B; C and D) Representative images from 2 patients and (E and F) a negative staining control. The original magnifications are noted at the upper left corner of each microphotograph. Scale bar, $100 \mu \mathrm{m}$.
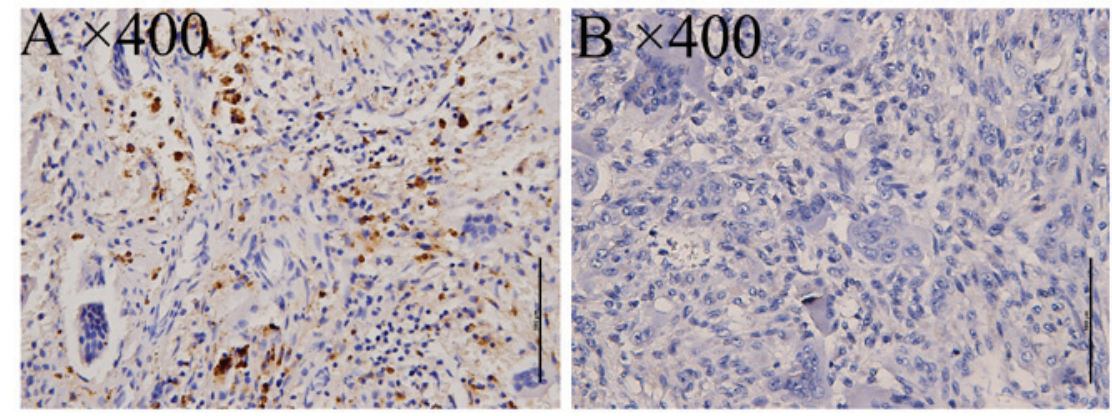

Figure 2. Immunohistochemical staining of mGluR4 in giant-cell tumor of bone. mGluR4 expression was detected as (A) cytoplasmic staining in tumor cells and (B) lack of such staining in a negative control. The original magnifications are noted at the upper left corner of each microphotograph. Scale bar, $100 \mu \mathrm{m}$.

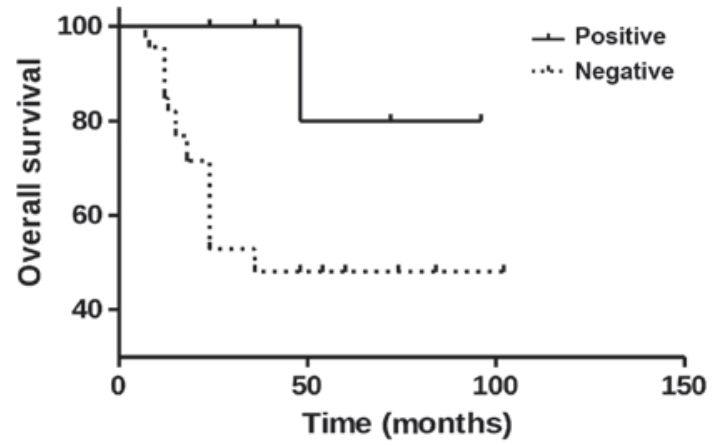

Figure 3. Survival analysis of osteosarcoma patients during a follow-up period of 12-102 months. The log-rank analysis revealed a higher survival rate in osteosarcoma patients with positive mGluR4 expression compared with those with negative mGluR4 expression $(\mathrm{P}=0.0122)$.

neuroprogenitors, as illustrated by positive mGluR4 staining, contributing to a favorable prognosis (11). Moreover, enhanced mGluR4 expression following PHCCC treatment was beneficial in medulloblastomas, and was found to be negatively correlated with neural tube cell tumor progression. Thus, the stimulation of mGlu4 expression may be used to treat Parkinson's disease in addition to medulloblastomas (12). Additionally, these findings suggest that mGluR4 may be considered as a phenotypic marker of medulloblastomas of lower malignant potential, such as the nodular desmoplastic histotype, or may negatively regulate tumor growth.

However, Chang et al (13) reported that mGluR4 overexpression may lead to 5 -fluorouracil tolerance, as this outcome was observed in 54\% of malignant colorectal carcinoma cases and was correlated with a poor prognosis. Another study investigating mGluR1/5/4 protein and gene expression in osteosarcomas reported varying expression levels among these receptors (14). While mGluR1 and mGluR5 were not statistically significant, mGluR4 was correlated with Enneking stage, tumor metastasis and poor prognosis in osteosarcoma patients.

According to our results, mGluR4 exhibited strong positive staining in cerebellar tissue, with a positive rate of $20.69 \%$ $(12 / 58)$ in osteosarcomas and $43.75 \%(14 / 32)$ in giant-cell tumors of bone, which is a benign tumor. Furthermore, tumors with elevated mGluR4 expression tended to be less agressive, suggesting a good prognosis. The statistical analysis 
revealed that mGluR4 expression is correlated with gender $(\mathrm{P}=0.0308)$, age $(\mathrm{P}=0.0489)$, Enneking stage $(\mathrm{P}=0.0415)$ and tumor volume $(\mathrm{P}=0.02)$ in osteosarcoma. The survival curves revealed a significantly higher survival rate in patients with positive mGluR4 expression compared with those with negative expression $(\mathrm{P}=0.0122)$. This correlation analysis was in accordance with our expectations, with the exception of gender. Due to the limited sample size, it would appear that gender is not really significant. A multivariate Cox regression analysis was performed and Enneking stage exerted a statistically significant effect on survival $(\mathrm{P}<0.01)$, suggesting that Enneking stage is the most important predictor of survival.

Glutamate signaling is involved in a wide variety of processes during normal bone formation, including cell differentiation and growth $(15,16)$. The concentration of glutamate is regulated by various types of cells in the bone environment (17). Since glutamate signaling plays an important role in maintaining skeletal homeostasis, it was hypothesized that this mechanism may stimulate metastatic tumor cells to differentiate, reducing the malignant potential $(10,11,18)$. A positive mGluR4 expression was found to be correlated with a positive prognosis and, therefore, may serve as a useful predictive indicator. While glutamate signaling appears to be promising, further molecular and genetic experimentation is required. While neoadjuvant chemotherapy has improved survival in patients with osteosarcoma, an effective targeted therapy is not yet available. Our findings taken together with those of previous studies indicate that the glutamate signaling pathway may serve as an important therapeutic target for osteosarcomas (19). However, further investigations are required to determine the potential of mGluR4 as a prognostic tool.

\section{References}

1. Ottaviani G and Jaffe N: The epidemiology of osteosarcoma. Cancer Treat Res 152: 3-13, 2009.

2. Savage SA, Mirabello L, Wang Z, Gastier-Foster JM, Gorlick R, Khanna C, Flanagan AM, Tirabosco R, Andrulis IL, Wunder JS, et al: Genome-wide association study identifies two susceptibility loci for osteosarcoma. Nat Genet 45: 799-803, 2013.

3. Ohtani Y, Harada T, Funasaka Y, Nakao K, Takahara C, Abdel-Daim M, Sakai N, Saito N, Nishigori C and Aiba A: Metabotropic glutamate receptor subtype-1 is essential for in vivo growth of melanoma. Oncogene 27: 7162-7170, 2008

4. Mathiesen JM, Svendsen N, Bräuner-Osborne H, Thomsen C and Ramirez MT: Positive allosteric modulation of the human metabotropic glutamate receptor 4 (hmGluR4) by SIB-1893 and MPEP. Br J Pharmacol 138: 1026-1030, 2003.

5. Teh J and Chen S: mGlu receptors and cancerous growth. Wiley Interdiscip Rev Membre Transp Signal 1: 211-220, 2012.
6. Ye ZC and Sontheimer H: Glioma cells release excitotoxic concentrations of glutamate. Cancer Res 59: 4383-4391, 1999

7. Ryo Y, Miyawaki A, Furuichi T and Mikoshiba K: Expression of the metabotropic glutamate receptor mGluR1 alpha and the ionotropic glutamate receptor GluR1 in the brain during the postnatal development of normal mouse and in the cerebellum from mutant mice. J Neurosci Res 36: 19-32, 1993.

8. Dorsam RT and Gutkind JS: G-protein-coupled receptors and cancer. Nat Rev Cancer 7: 79-94, 2007.

9. Brocke KS, Staufner C, Luksch H, Geiger KD, Stepulak A Marzahn J, Schackert G, Temme A and Ikonomidou C: Glutamate receptors in pediatric tumors of the central nervous system. Cancer Biol Ther 9: 455-468, 2010.

10. Canudas AM, Di Giorgi-Gerevini V, Iacovelli L, Nano G, D'Onofrio M, Arcella A, Giangaspero F, Busceti C, Ricci-Vitiani L, Battaglia G, et al: PHCCC, a specific enhancer of type 4 metabotropic glutamate receptors, reduces proliferation and promotes differentiation of cerebellar granule cell neuroprecursors. J Neurosci 24: 10343-10352, 2004.

11. Iacovelli L, Arcella A, Battaglia G, Pazzaglia S, Aronica E, Spinsanti P, Caruso A, De Smaele E, Saran A, Gulino A, et al: Pharmacological activation of mGlu4 metabotropic glutamate receptors inhibits the growth of medulloblastomas. J Neurosci 26: 8388-8397, 2006

12. Le Poul E, Boléa C, Girard F, Poli S, Charvin D, Campo B, Bortoli J, Bessif A, Luo B, Koser AJ, et al: A potent and selective metabotropic glutamate receptor 4 positive allosteric modulator improves movement in rodent models of Parkinson's disease. J Pharmacol Exp Ther 343: 167-177, 2012.

13. Chang HJ, Yoo BC, Lim SB, Jeong SY, Kim WH and Park JG: Metabotropic gluamate receptor 4 expression in colorectal carcinoma and its prognostic significance. Clin Cancer Res 11: 3288-3295, 2005.

14. Yang W, Maolin H, Jinmin Z and Zhe W: High expression of metabotropic glutamate receptor 4: Correlation with clinicopathologic characteristics and prognosis of osteosarcoma. J Cancer Res Clin Oncol 140: 419-426, 2014.

15. Spencer GL, McGrath CJ and Genever PG: Current perspectives on NMDA-type glutamate signaling in bone. Int J Biochem Cell Biol 39: 1089-1104, 2007.

16. Stepulak A, Luksch H, Gebhardt C, Uckermann O, Marzahn J, Sifringer M, Rzeski W, Staufner C, Brocke KS, Turski L and Ikonomidou C: Expression of glutamate receptor subunits in human cancers. Histochem Cell Biol 132: 435-445, 2009.

17. Takarada-Iemata M, Takarada T, Nakamura Y, Nakatani E, Hori $\mathrm{O}$ and Yoneda Y: Glutamate preferentially suppresses osteoblastogenesis than adipogenesis through the cystine/glutamate antiporter in mesenchymal stem cells. J Cell Physiol 226: 652-665, 2011.

18. Seidlitz EP, Sharma MK and Singh G: Extracellular glutamate alters mature osteoclast and osteoblast functions. Can J Physiol Pharmacol 88: 929-936, 2010.

19. Seidlitz EP, Sharma MK, Saikali Z, Ghert M and Singh G: Cancer cell line release glutamate into the extracellular environment. Clin Exp Metastasis 26: 781-787, 2009. 